\title{
Study on Double-sided Impedance Matching of Spherical Focusing Transducer in Ultrasonic Abrasive Flow Polishing System
}

\section{Li Wei}

Suzhou University of Science and Technology

Hua Li ( $\square$ lihua@mail.usts.edu.cn )

Suzhou University of Science and Technology and Suzhou Key Laboratory of Precision and Efficient Processing Technology

Dong Wei

Suzhou University of Science and Technology

\section{Research Article}

Keywords: focusing transducer, impedance matching, focus sonic pressure, abrasive flow polishing

Posted Date: August 10th, 2021

DOI: https://doi.org/10.21203/rs.3.rs-793725/v1

License: (c) (i) This work is licensed under a Creative Commons Attribution 4.0 International License. Read Full License

Version of Record: A version of this preprint was published at The International Journal of Advanced Manufacturing Technology on March 3rd, 2022. See the published version at https://doi.org/10.1007/s00170-022-08972-0. 


\title{
Study on double-sided impedance matching of spherical focusing transducer in ultrasonic abrasive flow polishing
}

\author{
system \\ WEI Li ${ }^{1}$, LI Hua ${ }^{1,2}$, WEI Dong ${ }^{1}$ \\ (1.School of Mechanical Engineering, Suzhou University of Science and Technology, Suzhou 215009, China; \\ 2. Suzhou Key Laboratory of Precision and Efficient Processing Technology, Suzhou 215009, China)
}

\begin{abstract}
The impedance matching of ultrasonic focusing spherical transducer is one of the primary factors affecting its work efficiency in ultrasonic focusing flow polishing system. To realize the efficient utilization of the vibration energy of the transducer, there are different requirements for the front and rear matching structure of the transducer. That is, the front matching structure should have high transmittance, and the rear matching structure should have high reflectivity. Based on the acoustic impedance matching theory and Kirchhoff's theory, the Influence of the front \& back impedance matching structure of spherical focusing ultrasonic transducer to the focus sound pressure is studied theoretically and experimentally. The new matching structure for spherical focusing transducer is proposed based on above research. The experimental result shows that the focus sonic pressure with the new matching structure is increased by $72.03 \%$ compared with the traditional structure.
\end{abstract}

Key words: focusing transducer; impedance matching; focus sonic pressure; abrasive flow polishing

\section{0 introduction}

Focused ultrasonic technology has been widely used in the fields of biomedicine, non-destructive testing, and ultrasonic imaging because of its advantages such as good directivity and high work efficiency. In recent years, this technology has also been applied in the precision machining of difficult-to-machine materials ${ }^{[1]}$. With focused ultrasonic technology the ultrasonic vibration energy can be effectively used in the efficiently and precisely material remove on the specified spot of workpiece surface. Therefore, it has a broad application prospects in industrial fields such as surface processing and polishing. There are three types of focused ultrasonic transducers: concave spherical self-focused, acoustic lens focused and array focused types ${ }^{[2]}$. The spherical self-focused ultrasonic transducer is a commonly used transducer, which activates the point energy at the focal point through self-focusing of the spherical structure to complete the processing work. The focus efficiency of the transducer is one primary factor affecting its working performance. In the field of mechanical processing, the high pressure generated by the vibration and cavitation effect pushes the abrasive flow to remove material on the workpiece surface.

The principle of focused ultrasonic processing is shown in Fig. 1. The piezoelectric ceramic plate vibrates in the direction of thickness and radiates sound waves to the concave (front) and convex (back) surfaces of the transducer simultaneously under the driven by ultrasonic power supplier. The front acoustic wave energy transmits and focuses at focus point through the medium to process the work surface. The back acoustic wave dissipates in the medium. To realize the efficient utilization of the vibration energy of the transducer, there are different requirements for the front and rear matching structure of the transducer. That is, the front matching structure should have high transmittance, and the rear matching structure should have high reflectivity. Many scholars at home

About author: Wei Li (1997-), male.

Corresponding author: Li Hua, Professor. E-mail: lihua@mail.usts.edu.cn 
and abroad have done a lot of research on the structure of different transducers. In the field of ultrasonic testing transducers, Toda Minoru et al. ${ }^{[3]}$ studied the effect of multi-layer polymer matching layers and metal backing on the transducer; $\mathrm{Fu} \mathrm{Lin}{ }^{[4]}$ et al. studied the effections of

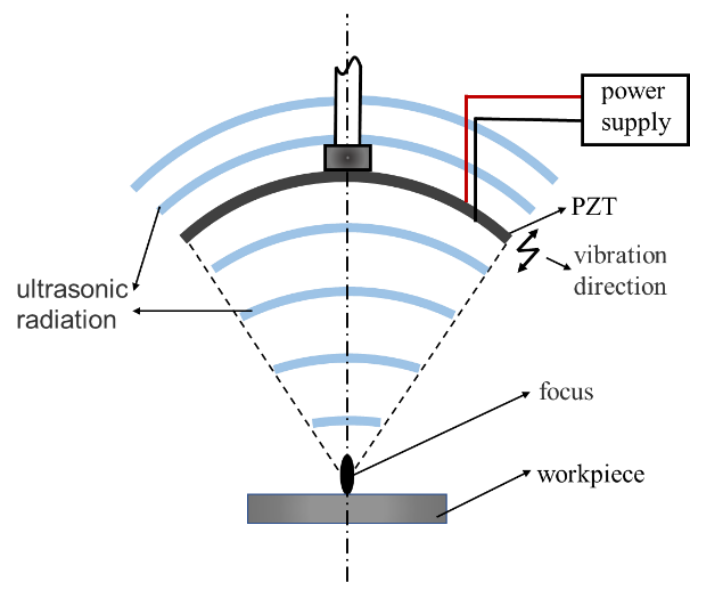

Fig. 1 Principle of focused ultrasonic processing

different backing thickness on the transducer to acoustic impedance and mechanical loss factor, provided a theoretical basis for the design of ultrasonic transducers. This backing structure is used to absorb aftershock signals to improve the ultrasonic detection accuracy. In the field of ultrasonic radiation transducers, Zhang Jiayu ${ }^{[5]}$ et al. simulated the radiation efficiency of planar piezoelectric transducers under different backing materials, finding that the air backing layer had a better radiation effect. Liu Kai ${ }^{[6]}$ et al. found in structural simulation design of the front matching layer of the spherical focused transducer that, the sound pressure and sound intensity at the focus of the transducer were both enhanced after addition of the matching layer. But there is no systematic study of focusing spherical transducers in these studies.

To optimize the structure of the focusing spherical ultrasonic transducer and achieve highefficiency energy output, this paper systematically studies the matching parameters of the focusing spherical transducer, analyzes the principle of front and back matching. The matching structure transducer is studied based on numerical theoretical calculation and sound field simulation, and these results were verified by sound pressure measurement experiments.

\section{Theoretical analysis on matching of focused transducer}

The sound pressure at each point in the radiation sound field of the focused ultrasonic transducer can be calculated based on the continuous wave theory ${ }^{[7]}$, as shown in Fig. 2.

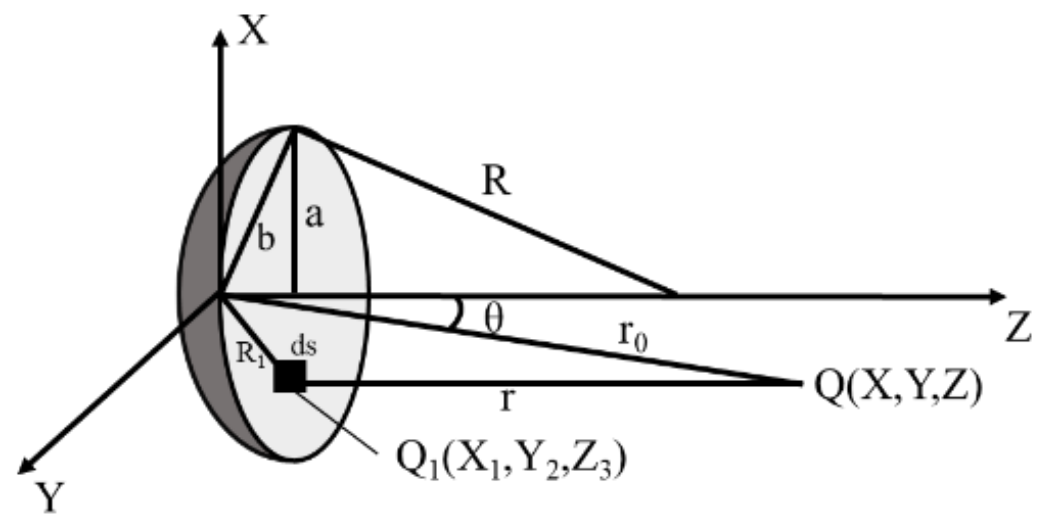

Fig. 2 The calculation coordinates of the sound pressure of the concave spherical transducer 
In the Fig.2, $\mathrm{R}$ is the radius of curvature of the transducer, a is the opening radius of the spherical shell, $\mathrm{b}$ is the distance from the origin to the sphere edge, $\mathrm{r}_{0}$ is the distance from the origin of the coordinates to a point $Q$ in the sound field, and $\theta$ is the intersection angle between $r_{0}$ and $Z$ axis; ds is the unit area on the radiating surface; $R_{1}$ is the distance from the origin of the coordinates to the radiating surface; $r$ is the distance from point $Q_{1}$ to $Q$ on the radiating surface. Then, the sound pressure at point $\mathrm{Q}$ is:

$$
\begin{gathered}
r=\left[r_{0}^{2}-2 r_{0} R_{1} \sqrt{1-\frac{R_{1}^{2}}{4 R^{2}}} \sin \theta \cos \varphi+\right. \\
\left.R_{1}^{2}\left(1+\frac{r_{0} \cos \theta}{R}\right)\right]^{\frac{1}{2}} \\
p\left(r_{0}, \theta\right)=i f \rho u_{0} \int_{0}^{2 \pi} \int_{0}^{b} \frac{e^{-j k r}}{r} R_{1} \mathrm{~d} R_{1} \mathrm{~d} \varphi
\end{gathered}
$$

According to Huygens' theory, the sound pressure $p$ in the $\mathrm{Z}$ direction of the acoustic axis can be obtained as follows ${ }^{[8]}$ :

$$
p=i f \rho u_{0} \cdot \frac{2 \pi^{2} a^{2}}{R \lambda} \cdot \frac{\sin \left[\pi \frac{a^{2}}{2 z \lambda}\left(\frac{z}{R}-1\right)\right]}{\pi \frac{a^{2}}{2 z \lambda}\left(\frac{z}{R}-1\right)}
$$

Where: $f$ is the ultrasonic vibration frequency; $\rho$ is the medium density; $\mathrm{u}_{0}=\mathrm{A} / \omega ; \mathrm{A}$ is the ultrasonic vibration amplitude; $\omega=2 \pi f, \lambda=\mathrm{c} / f$; $\mathrm{c}$ is the propagation speed of sound waves in the medium.

The transmission of sound waves in the medium is shown in Fig. 3. The transmittance $T_{3}$ is affected by the thickness $d$ of the middle layer II ${ }^{[10]}$ :

$$
T_{3}=\frac{4 Z_{1} Z_{3}}{\left(Z_{1}+Z_{3}\right)^{2} \cos \frac{2 \pi d}{\lambda_{2}}+\left(Z_{2}+\frac{Z_{1} Z_{3}}{Z_{2}}\right)^{2} \sin ^{2} \frac{2 \pi d}{\lambda_{2}}}
$$

In the formula, $Z_{1}, Z_{2}, Z_{3}$ are the acoustic impedances of medium I, II, and III respectively, $Z_{i}=\rho_{i} c_{i}$; $\lambda_{2}=c_{2} / f$ is the wavelength in medium II; $c_{2}$ is the sound velocity of medium II; $f$ is resonance frequency.

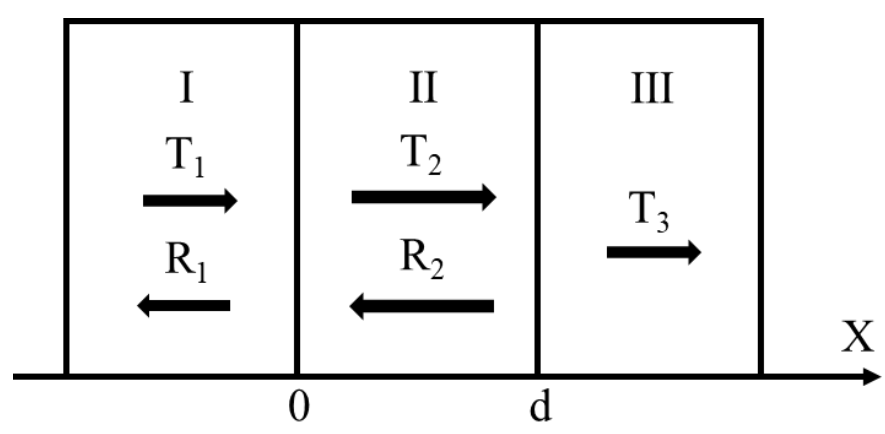

Fig. 3 The reflection and transmission of sound waves in a three-layer medium

\subsection{Front matching}

In focused ultrasound abrasive flow processing, water based abrasive flow is usually used as the working medium.as the acoustic impedance difference between the transducer and the transmission medium (abrasive flow), the acoustic wave reflects at interface between water and piezoelectric transducer, which will increase the sound energy loss ,reduce the working efficiency of the focused ultrasonic system and cause failure of the transducer.

According to the acoustic impedance matching theory ${ }^{[9]}$, the front matching layer of the 
transducer can effectively reduce the acoustic impedance mismatch during operation. In practical application there are two kinds of front matching layer structure as shown in Fig. 4. One is called flat matching layer, and the other is concave matching layer.

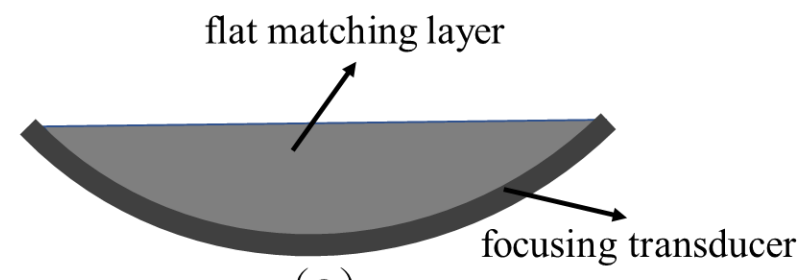

(a)

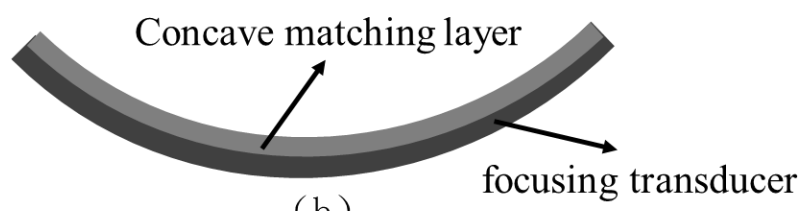

(b)

Fig. 4 structure type of front matching layer

The sound pressure distribution on the axis of the focused transducer can be numerically calculated without considering the attenuation of sound energy in the medium for these two matching structures. The material parameters used in the calculation are shown in Table 1.

\begin{tabular}{lll}
\multicolumn{3}{c}{ Table 1 Material parameter } \\
\hline Medium & Density $\mathrm{kg} / \mathrm{m}^{3}$ & Sound velocity $\mathrm{m} / \mathrm{s}$ \\
\hline I (PZT) & 7500 & 4000 \\
II (silica gel) & 3095 & 1100 \\
III (water) & 1000 & 1480 \\
\hline
\end{tabular}

\subsubsection{Concave matching layer}

The concave matching layer is of equal thickness on the concave surface of the transducer, so it can be directly calculated by formula (1) (2), and the sound pressure $\mathrm{P}_{0}$ on the axis is:

$$
P_{0}=p \cdot T_{3}=i f \rho u_{0} \cdot \frac{2 \pi^{2} a^{2}}{R \lambda} \cdot \frac{\sin \left[\pi \frac{a^{2}}{2 z \lambda}\left(\frac{Z}{R}-1\right)\right]}{\pi \frac{a^{2}}{2 z \lambda}\left(\frac{Z}{R}-1\right)} \cdot \frac{4 Z_{1} Z_{3}}{\left(Z_{1}+Z_{3}\right)^{2} \cos \frac{2 \pi d}{\lambda_{2}}+\left(Z_{2}+\frac{Z_{1} Z_{3}}{Z_{2}}\right)^{2} \sin ^{2} \frac{2 \pi d}{\lambda_{2}}}
$$

The calculation results are shown in Fig. 5. From which it can be seen that the sound pressure at the focus is greatly increased and changes with thickness of the matching layer. The maximum sound pressure is got when the thickness of the concave matching layer is equal to a quarter wavelength. 


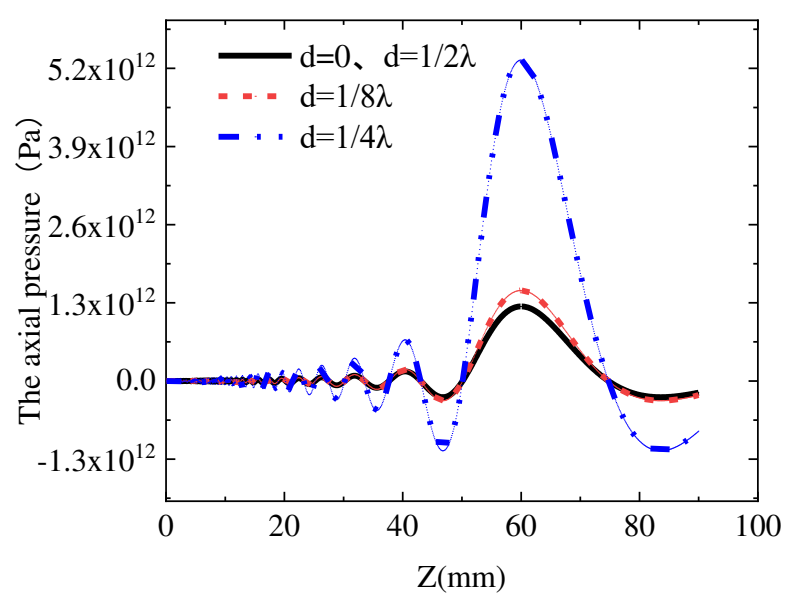

Fig. 5 Theoretical curves of sound pressure on the axis under different matching layer thicknesses

$$
f=1 \mathrm{MHz}, \mathrm{A}=10^{-5} \mathrm{~m}, \rho=10^{3} \mathrm{~kg} / \mathrm{m}^{3}, \mathrm{a}=0.03 \mathrm{~m}, \mathrm{R}=0.06 \mathrm{~m}
$$

\subsubsection{Flat matching layer}

For the flat matching layer, as the thickness of the flat matched layer is not evenly distributed on the spherical transducer, the sound pressure on the axis should be calculated according to the principle of sound field superposition. As shown in Fig. 6 and 7. where $\mathrm{H}$ is the axial thickness of the matching layer.

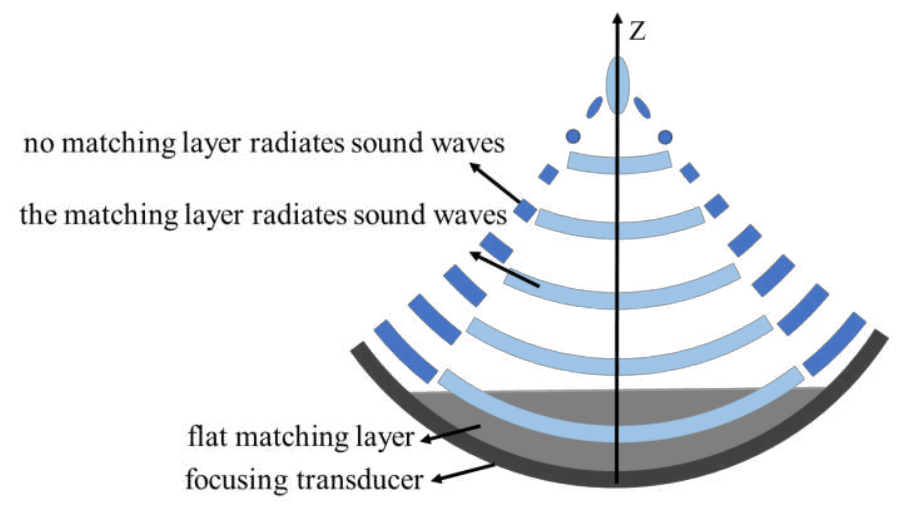

Fig. 6 Sound field superposition model

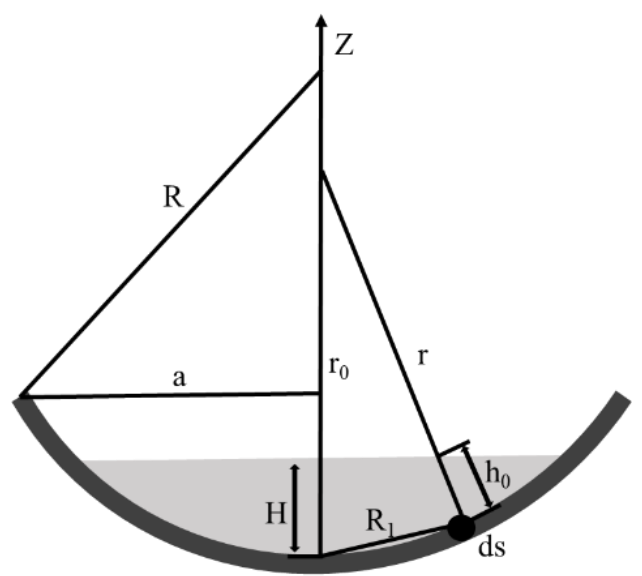

Fig. 7 Calculation model of matching layer thickness 
When $\theta=0^{\circ}$, that is, $r=\left[r_{0}^{2}+R_{1}^{2}\left(1+\frac{r_{0} \cos \theta}{R}\right)\right]^{\frac{1}{2}}$, the sound pressure distribution of focused transducer on the axis can be calculated, as shown in Fig. 7. At this time, the transmittance $T_{3}{ }^{\prime}$ changes with the change of $r_{0}$. By geometric calculation, there is:

$$
\begin{gathered}
T_{3}{ }^{\prime}=\frac{4 Z_{1} Z_{3}}{\left(Z_{1}+Z_{3}\right)^{2} \cos \frac{2 \pi H}{\lambda_{2}}+\left(Z_{2}+\frac{Z_{1} Z_{3}}{Z_{2}}\right)^{2} \sin ^{2} \frac{2 \pi H}{\lambda_{2}}} \\
H=\sqrt{r_{0}^{2}+R_{1}^{2}\left(\frac{r_{0}}{R}+1\right)}-\sqrt{\left(h-r_{0}\right)^{2}+\frac{\left(h-r_{0}\right)^{2}\left(R_{1}^{2}-\frac{R_{1}^{4}}{4 r_{0}^{2}}\right)}{r_{0}^{2}-R_{1}^{2}+\frac{R_{1}^{4}}{4 r_{0}^{2}+R_{1}^{2}\left(\frac{r_{0}}{R}+1\right)}}}
\end{gathered}
$$

Therefore, the sound pressure of the matching layer covering part on the axis is:

$$
P=i f \rho u_{0} \int_{0}^{2 \pi} \int_{0}^{b_{0}} \frac{e^{-j k r}}{r} R_{1} \cdot T_{3}{ }^{\prime} \mathrm{d} R_{1} \mathrm{~d} \varphi
$$

Then, the sound pressure $\mathrm{P}$ at any point can be calculated as follows:

$$
P=i f \rho u_{0} \int_{0}^{2 \pi} \int_{0}^{b_{0}} \frac{e^{-j k r}}{r} R_{1} \cdot T_{3}{ }^{\prime} \mathrm{d} R_{1} \mathrm{~d} \varphi+i f \rho u_{0} \int_{0}^{2 \pi} \int_{b_{0}}^{b} \frac{e^{-j k r}}{r} R_{1} \mathrm{~d} R_{1} \mathrm{~d} \varphi \cdot T_{3}(\mathrm{~d}=0)
$$

The relationship between the axial sound pressure and the flat matching layer thickness $\mathrm{H}$ can be obtained through numerical calculation software, as shown in Fig. 8.

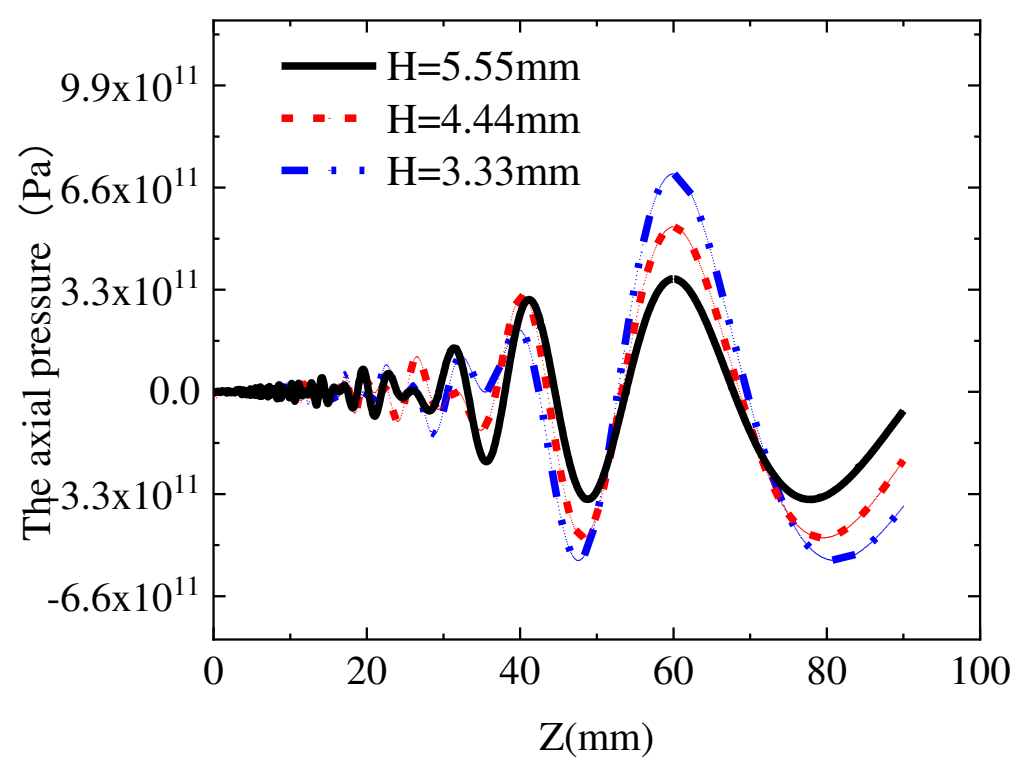

Fig. 8 The effect of flat matching layer thickness on sound pressure on the axis

It can be seen from the results that the sound pressure at focus is vary with thickness $\mathrm{H}$ of flat matching layer. For the same spherical focused ultrasonic transducer, the maximum focus acoustic pressure with the concave matching layer is obviously higher than that with the flat matching layer.

\subsection{Back Matching}




\subsubsection{Traditional backing structure}

Different from the front matching layer, the function of the back matching layer is to increase the reflectivity of the radiated sound waves on the back of the transducer and reduce the energy loss on the back. The traditional back matching structure is a cylinder (steel or aluminum) with concave end surface matching the back of the spherical transducer at one end. The steel cylinder and the spherical transducer are glued together as shown in Fig. 9.

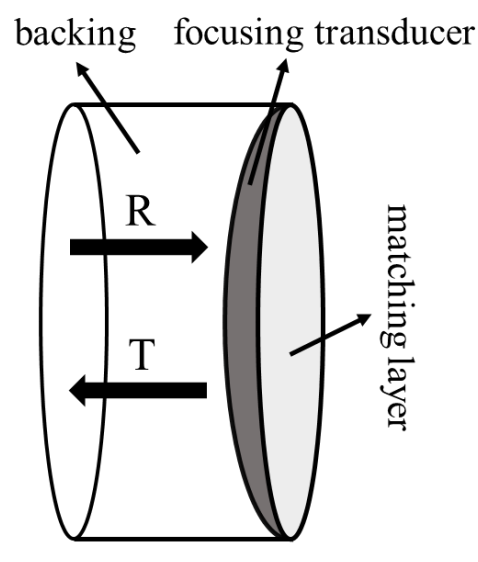

Fig. 9 Traditional backing structure model

The longitudinal wave reflectivity $\mathrm{R}$ for this structure can be calculated as follows ${ }^{[11]}$ :

$$
R=\frac{Z_{D}-Z_{A}}{Z_{D}+Z_{A}}
$$

where: $Z_{A}, Z_{D}$ are the impedance of the piezoelectric ceramic and the backing, respectively. Obviously, in the traditional backing structure, part of the sound wave $\mathrm{T}$ is still transmitted through the backing cylinder, leading to the loss of the sound energy.

\subsubsection{New multilayer structure}

In order to get higher sound wave reflective rate, A new back matching structure with three-layer medium is proposed as Fig.10. A medium layer (medium II ) with different impedance with transducer is inserted between the piezoelectric ceramic and the backing cylinder.

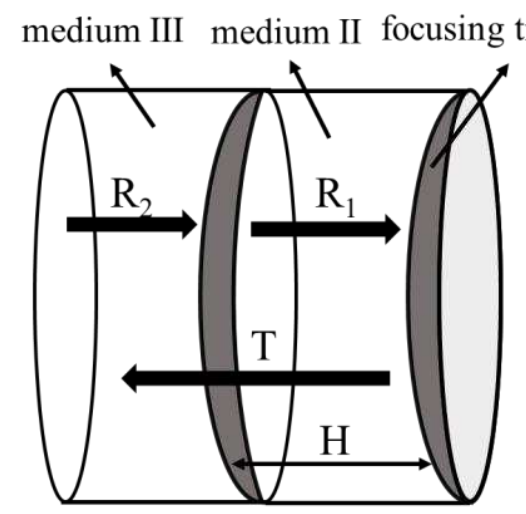

Fig. 10 Acoustic reflection model

The longitudinal wave reflectivity $\mathrm{R}_{1}$ can be calculated as follows ${ }^{[12]}$;

$$
R_{1}=\frac{e^{-2 \pi i H / \lambda_{B}}\left(Z_{A}+Z_{B}\right)\left(Z_{B}-Z_{C}\right)+e^{2 \pi i h / \lambda_{B}}\left(Z_{A}-Z_{B}\right)\left(Z_{B}+Z_{C}\right)}{e^{-2 \pi i H / \lambda_{B}}\left(Z_{B}-Z_{A}\right)\left(Z_{C}-Z_{B}\right)+e^{2 \pi i h / \lambda_{B}}\left(Z_{A}+Z_{B}\right)\left(Z_{B}+Z_{C}\right)}
$$

In the formula, $Z_{A}, Z_{B}, Z_{C}$ are the acoustic impedance of piezoelectric ceramics, medium II and medium III respectively; $\mathrm{H}$ is the thickness of medium II; $\lambda_{B}=c_{B} / f$ is the wavelength of medium 
II; " $f$ " is resonance frequency; $c_{B}$ is the sound velocity of medium II, and its value is determined by the type of material, as shown in Table 2 .

\begin{tabular}{|c|c|c|}
\hline Materials & Density $\mathrm{kg} / \mathrm{m}^{3}$ & Sound velocity $\mathrm{m} / \mathrm{s}$ \\
\hline steel & 7840 & 5941 \\
\hline air & 1.293 & 343 \\
\hline aluminum & 2700 & 6260 \\
\hline water & 1000 & 1480 \\
\hline
\end{tabular}

Therefore, the relationship curve between the sound pressure on the axis of the focused ultrasonic transducer and the reflectivity $R_{1}$ can be obtained through numerical simulation. It can be seen that the reflectivity reaches the maximum when the thickness of the medium II is an odd multiple of quarter wavelength. At the same time, the parameters of medium III also affect the reflectivity. The change law is shown in Fig. 11.

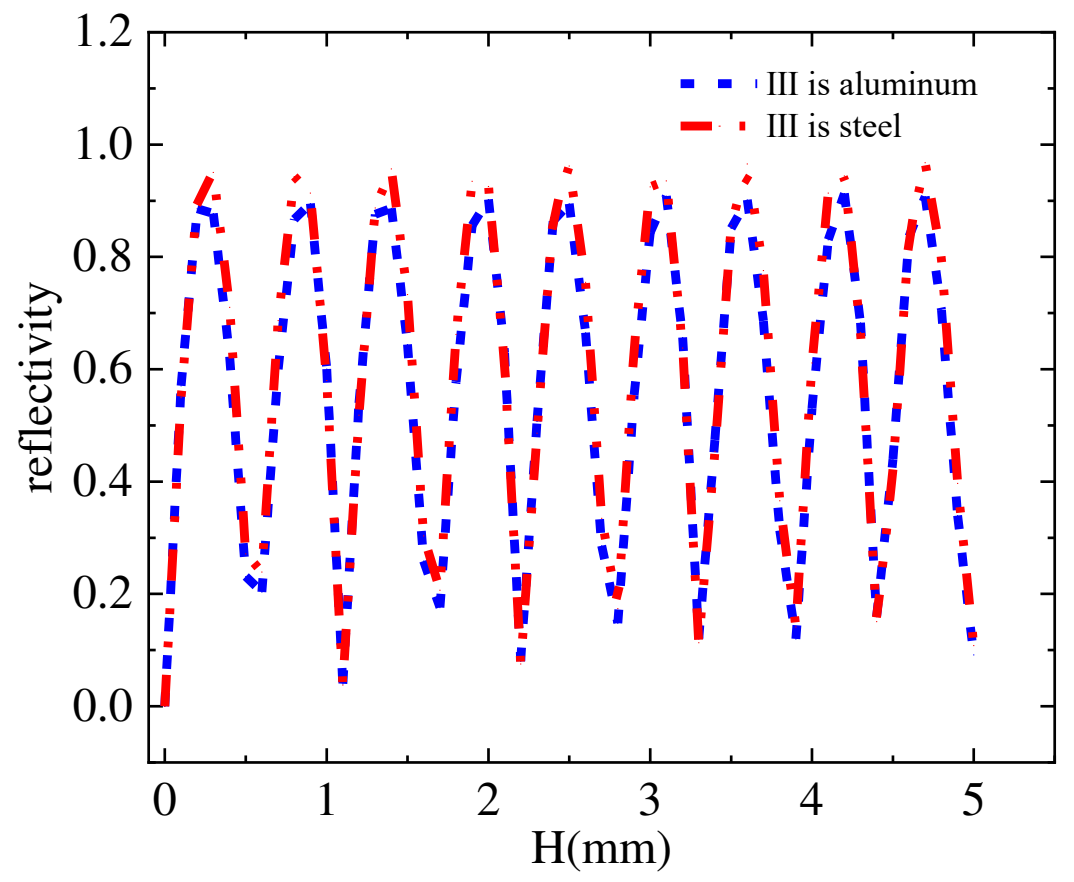

Fig. 11 The relationship between the thickness of medium II and reflectivity

It can be seen from above analyses that the three-layer reflection structure has significantly increased sound pressure at the focus compared with the traditional backing structure. The focus sound pressure reaches the maximum when the thickness of the intermediate layer (medium II) equal to the odd times of quarter wavelength.

In summary, it can be seen from the results of numerical calculations that the front and back acoustic matching of the focused ultrasonic transducer can reduce the sound energy loss and increase the sound utilization rate. However, accuracy of this result requires further demonstration.

\section{Sound field simulation}

Theoretical analysis shows that the matched structural parameters of the focused transducer have different effects on its radiation sound field. In order to verify the accuracy of theoretical analysis, the structure model of spherical focused ultrasonic transducer was established in the sound 
field simulation software.The simulation model is shown in Fig. 12.

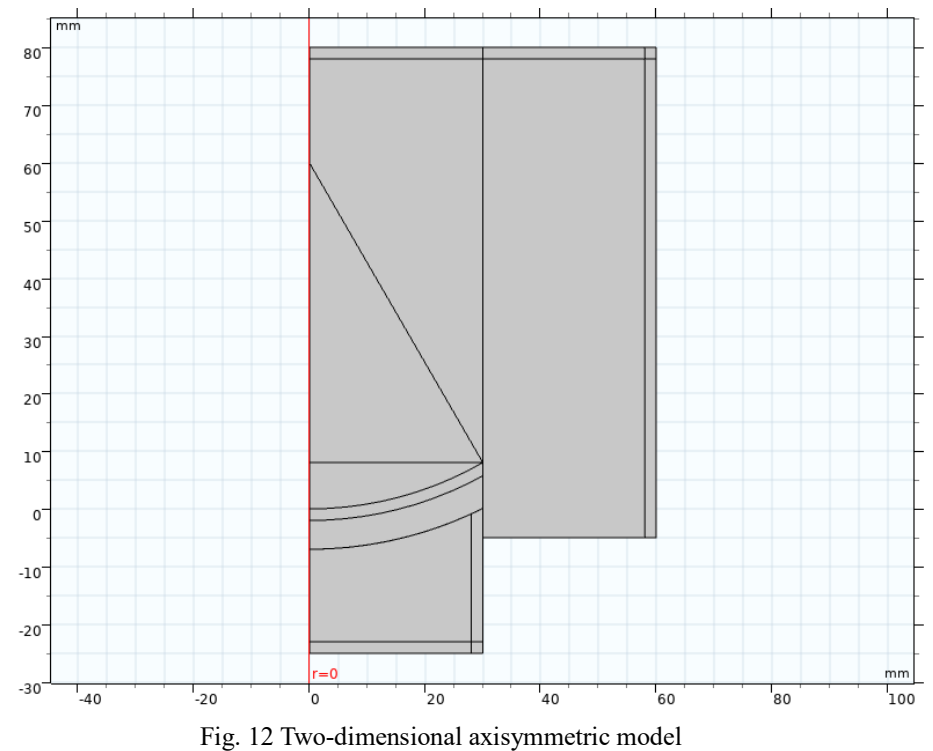

2.1 The effect of front matching structural parameters

The focusing effect of the transducer is different with the front matching structure. From the numerical calculation results, it can be seen that the thickness and structure of the front matching layer have a great influence to the focus sound pressure. Simulation is performed by changing its structural parameters, with its result shown in Fig. 14.

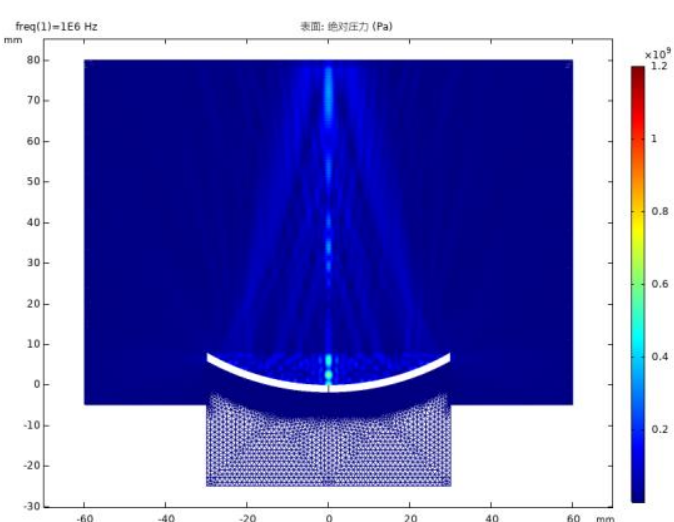

(a)

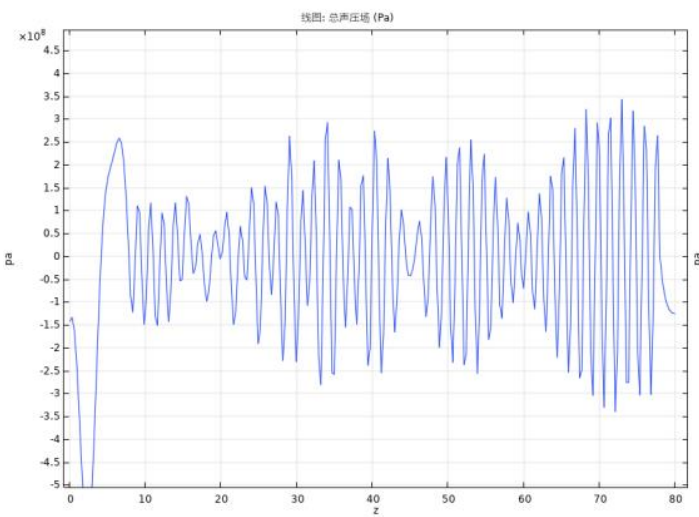

(b)

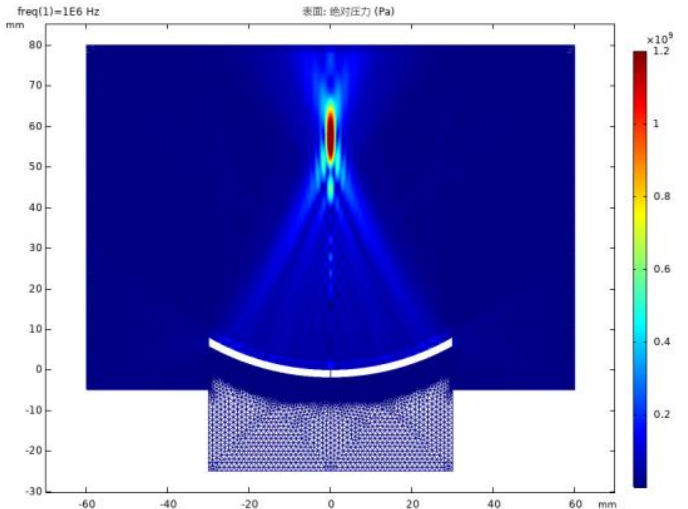

(b)

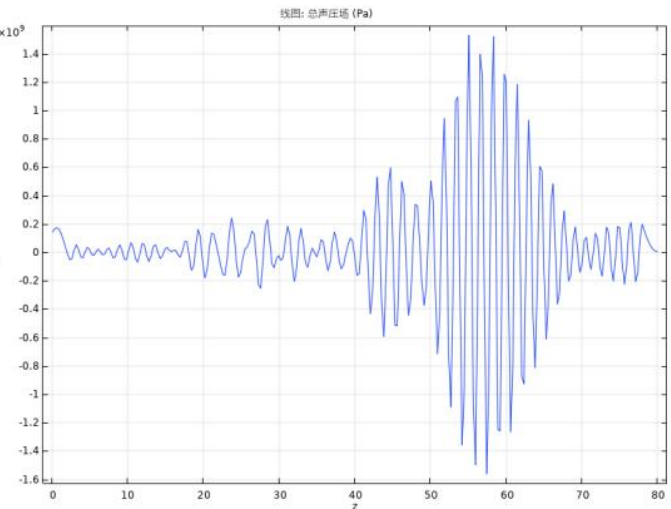

(d)

Fig. 14 Simulation results 
(A) Flat matching layer; (b) Concave matching layer; (c) sound pressure curve of flat matching layer; (d) sound pressure curve of concave matching layer

Compared with the flat matching layer, the concave matching layer has a better acoustic matching effect, and the sound pressure of the focusing transducer is significantly enhanced at the focal point. The sound pressure reaches the maximum at the focus when the matching layer thickness is an odd multiple of quarter wavelength, as shown in Fig. 15.

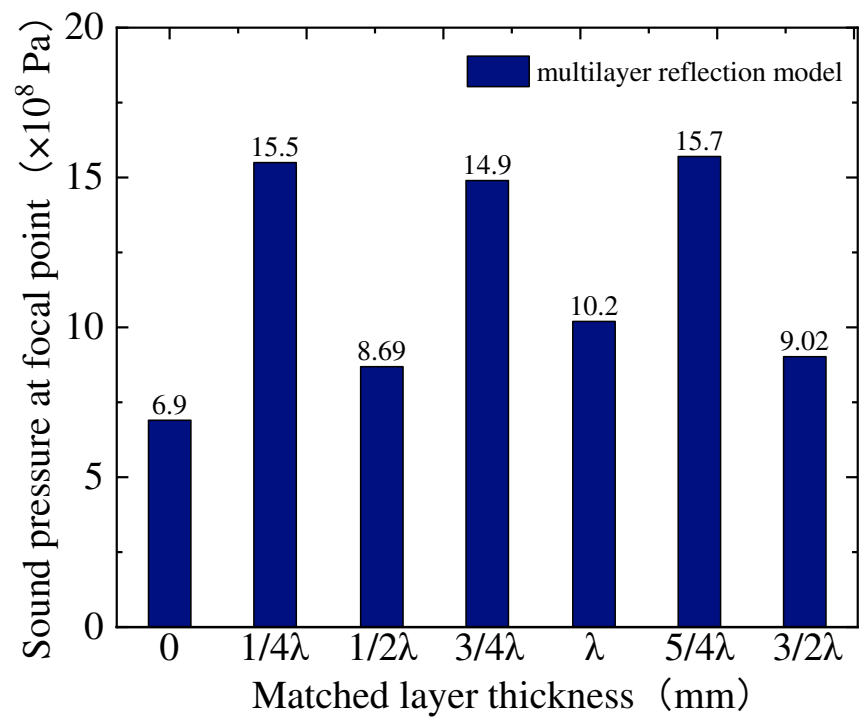

Fig. 15 Simulation results under different matching layer thicknesses

\subsection{The effect of back matching structure parameters}

The acoustic field simulation results of spherical focusing transducers under the two different post matching structures, the traditional backing structure and the new three layer reflection structure, are no different from those of the theoretical analysis. The simulation results are shown in Fig. 16.

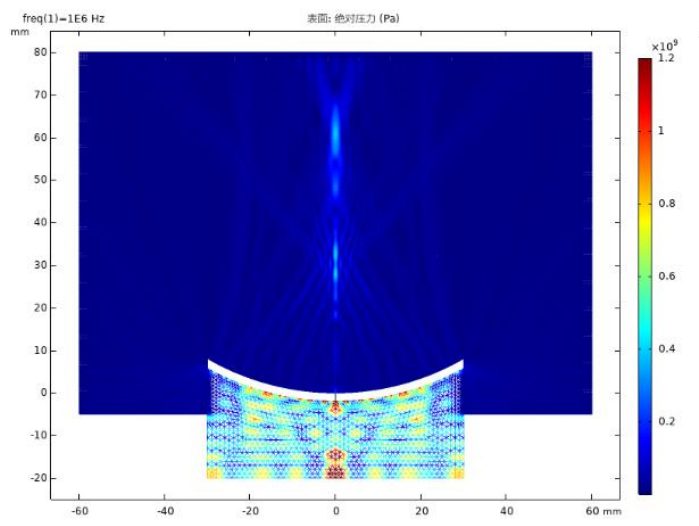

(a)

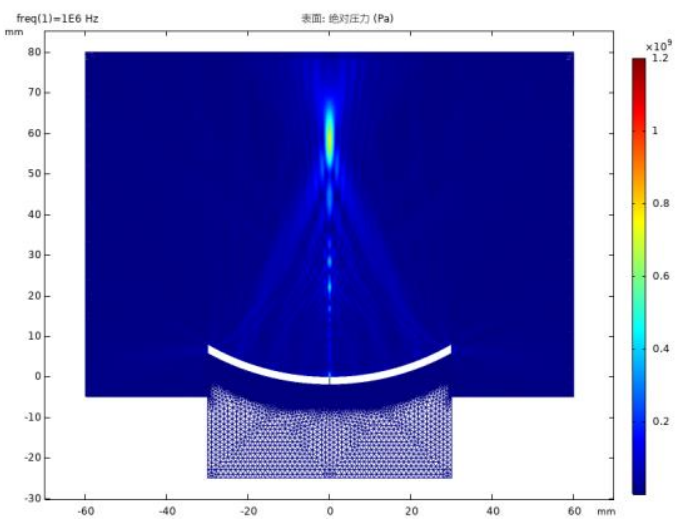

(b) 


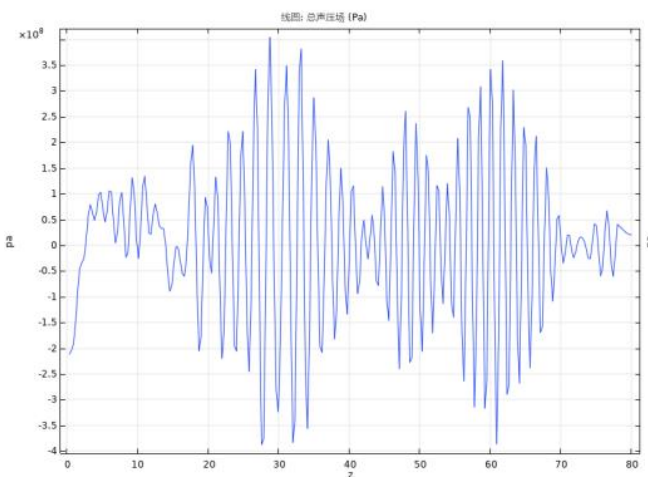

(c)

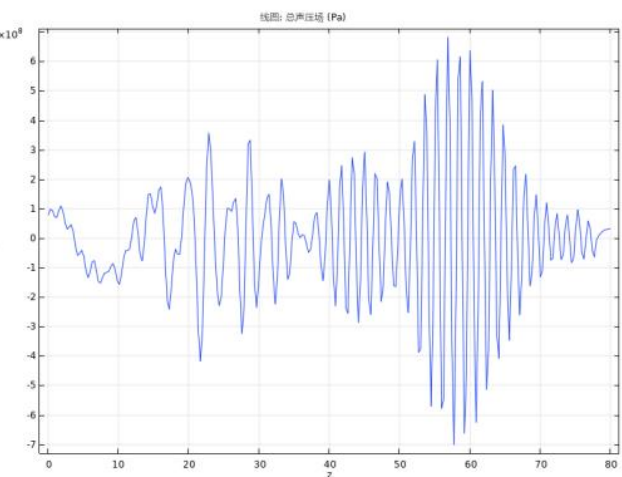

(d)

Fig.16 Simulation result

(A) Traditional backing structure; (b) Double reflection structure; (c) Sound pressure curve of flat traditional backing structure; (d) Sound pressure curve of acoustic reflection model

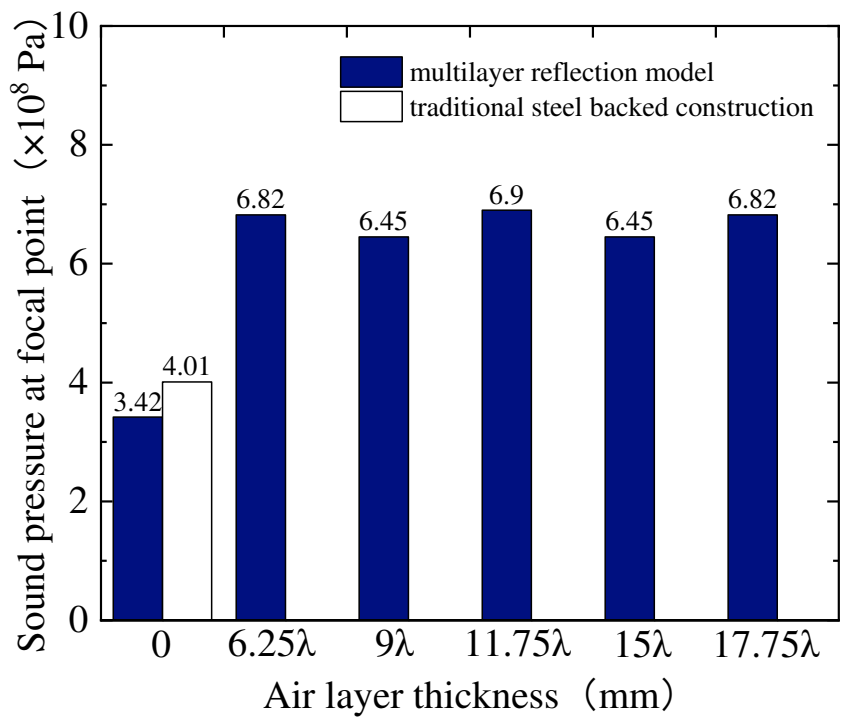

Fig. 17 Simulation results under different matching layer thicknesses

The effect of the air layer thickness of the sound pressure on the sound pressure is shown in Fig. 17. It can be seen from the Fig. that the acoustic impedance mismatch on the back of the spherical shell in the traditional structure makes most energy consumed in the backing. The energy loss in the double-layer reflective structure is significantly reduced, and the sound pressure at the focal point is greater than that of the traditional structure. The thickness of back matching layer does not bring obvious effect from the impedance parameter of the medium III (aluminum), and the focus sound pressure reaches the maximum when the thickness of the back matching layer equal to the odd times of quarter wavelength.

\section{Structural design and experimental research}

The results of numerical calculation and sound field simulation show that the sound utilization rate and the sound pressure at the focal point of the focused transducer can be increased by the reasonable front and back matching layer structure. Therefore, this paper designs a new type of focused ultrasonic transducer based on the front and back matching layer model, and its structure is shown in Fig. 18 and 19. 


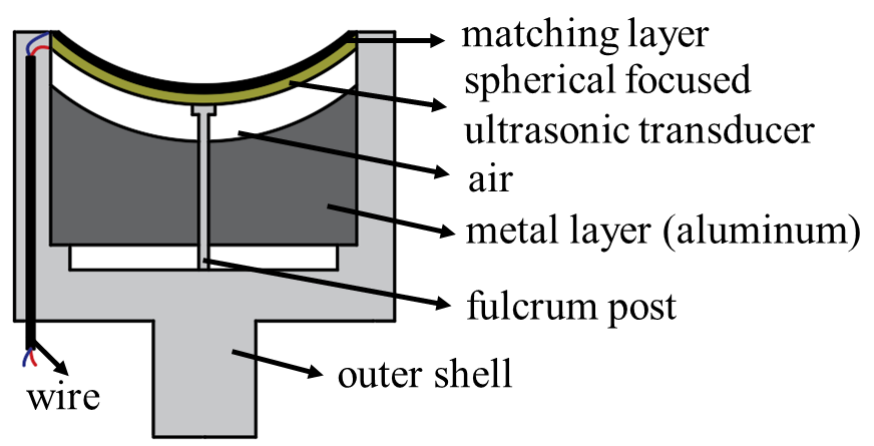

Fig. 18 The structure model of the new focused transducer

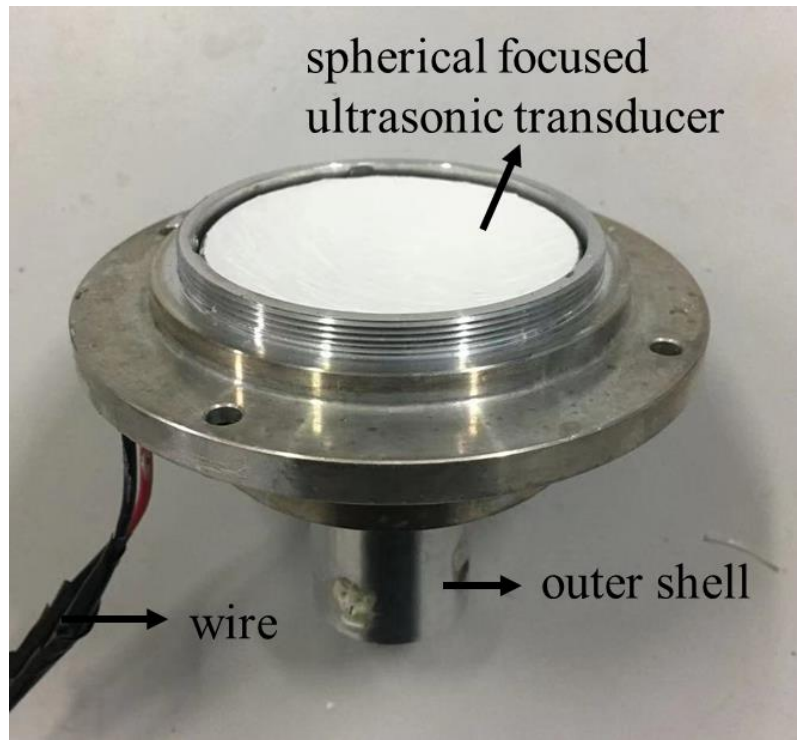

Fig. 19 Spherical focused transducer

In order to verify that the transducer of this structure can effectively increase the sound utilization rate and enlarge the sound pressure at the focal point, we measured and compared the sound pressure on the axis of the focused ultrasonic transducer in two different structures of the traditional backing structure and the new transducer structure.

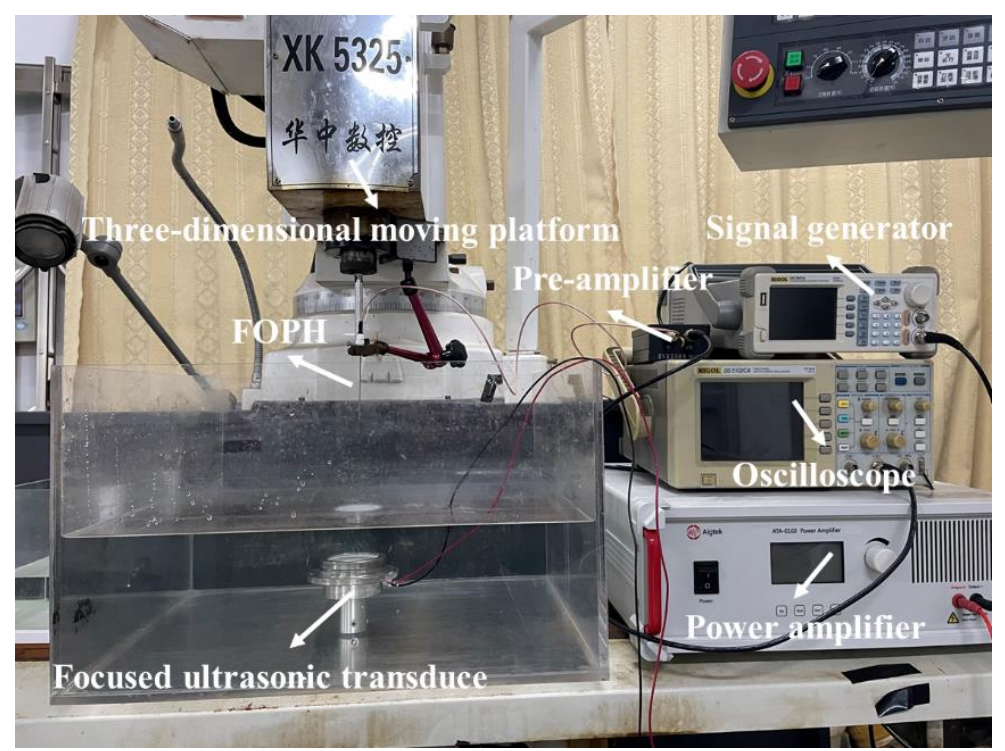

Fig. 20 Sound pressure measurement system 
The sound pressure measurement system is composed of an ultrasonic generator, a power amplifier, a digital oscilloscope, a probe hydrophone, a preamplifier, and a focused ultrasonic energy device, as shown in Fig. 20.The ultrasonic vibration frequency of the transducer is $1.2 \mathrm{MHz}$.The sound pressure signal is measured by the hydrophone and displayed on oscilloscope ,the peak-topeak voltage is recorded by the oscilloscope. The sound pressure $\mathrm{P}$ on the axis can be calculated.:

$$
P=10^{[(20 \times \lg U-M) / 20]}
$$

Where, $\mathrm{U}$ is the voltage value displayed by the oscilloscope, and the hydrophone sensitivity level is $\mathrm{M}=-228.9 \mathrm{~dB}$. The measurement results are shown in Fig. 21 .

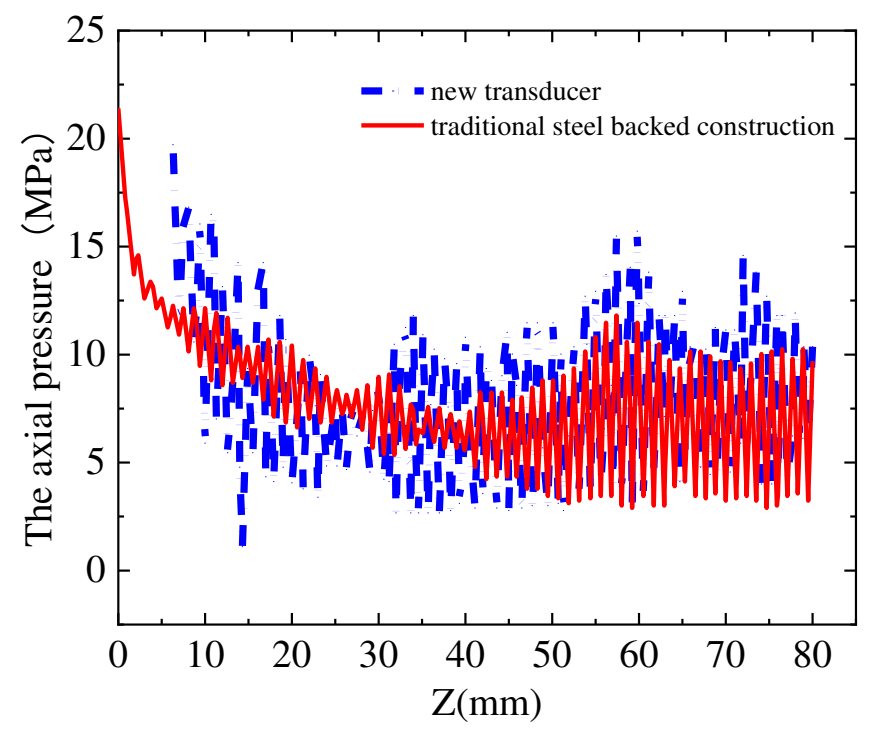

Fig. 21 Sound pressure curve according to experimental measurement

From the sound pressure distribution on the axis, it can be seen that the focused ultrasonic transducer in the new structure has higher sound pressure value in the focal region than the traditional backing structure (without matching layer), and the maximum sound pressure of the new structure reaches $15.3 \mathrm{MPa}$ near the focal point, an increase of $42.3 \%$ compared to the traditional steel backing structure.

Finally, a front matching layer (single-component room temperature cured silica gel) with a thickness amounting to an odd multiple of quarter wavelength is added to the focused spherical shell, and the sound pressure value on the axis of the focused transducer of the new structure is measured. The results showed that after the front matching layer was installed, the sound pressure value near the focal point increased significantly, with the maximum sound pressure reaching $20.3 \mathrm{MPa}$, this is an increase of $32.7 \%$ compared to the previous front matching layer. The specific values are shown in Fig. 22. 


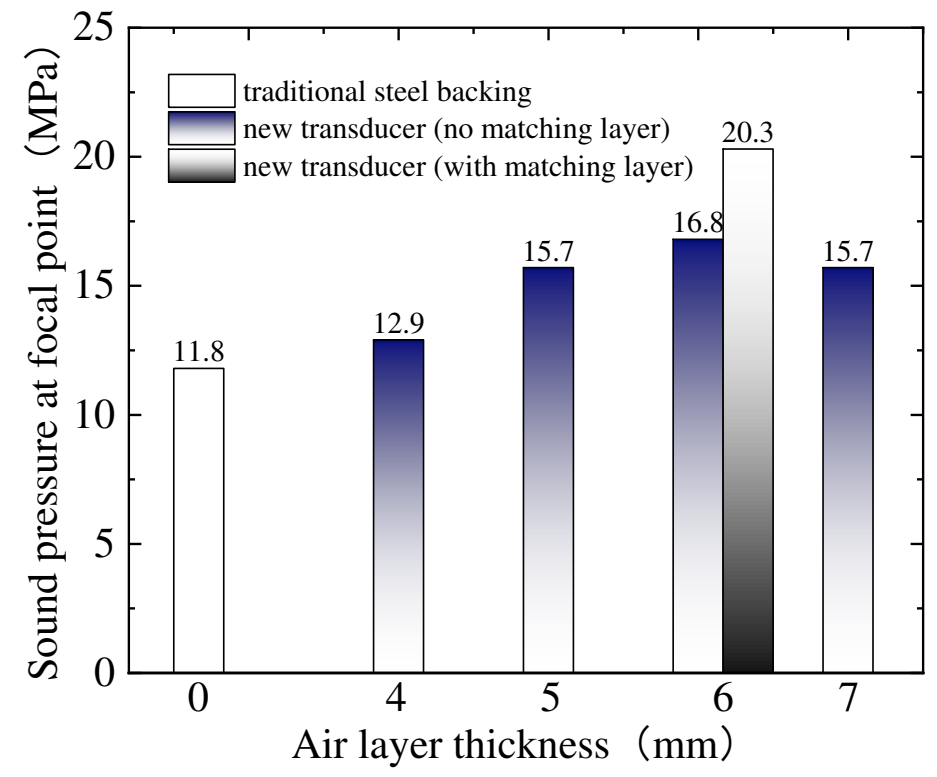

Fig. .22 Sound pressure measurement values

\section{Conclusion}

(1) The front matching layer of the focused ultrasonic transducer can increase the transmission efficiency of sound waves in the medium. From the numerical calculation and simulation results, it can be known that when the thickness of the current matching layer is an odd multiple of quarter wavelength, the sound pressure at the focal point of the transducer reaches the maximum.

(2) The back matching of the focused ultrasonic transducer can also improve the sound utilization rate and increase the sound pressure. Through numerical simulation and sound field simulation, it can be known that when the thickness of the intermediate layer is an odd multiple of quarter wavelength, there is maximum sound pressure increase at the focal point.

(3) A new type of focused ultrasonic transducer was designed and the axial sound pressure was measured. From the experimental results, it can be known that the maximum focus sound pressure of the focused transducer in the new structure is increased by $29.7 \%$ compared with the traditional structure. After adding of the front matching layer, the sonic pressure is increased by $32.7 \%$ compared to the previous front matching layer.

\section{Reference}

[1] LI Hua, REN Kun, et al.Ultrasonic vibration aided abrasive flow polishing technology research review [J/OL]. Journal of mechanical engineering: 1-21 [2021-04-28].

[2] Wu Yan, Fei Chunlong et al.Research Status and Development of Focused Ultrasonic Transducers [J]. Piezoelectrics \& Acousto-optics,2019,41(06):904-909.

[3] Toda Minoru,Thompson Mitch. Detailed investigations of polymer/metal multilayer matching layer and backing absorber structures for wideband ultrasonic transducers.[J]. IEEE transactions on ultrasonics, ferroelectrics, and frequency control, 2012,59(2).

[4] Fu Lin, Gao Yongkang, Gao Jingmin.Influence of Backlining Parameters on the Characteristics of Thickness Molded Electric Transducer[J]. Acta Acoustica Sinica,2019,44(2):251-257..

[5] Ya hang Jiayu, Yin Guanjun, Guo Jianzhong. Research on the influence of backing on the radiation efficiency 
of ultrasonic transducer in water[A]. 2019 Shanghai - Xi 'an Acoustical Society 6th Acoustics Academic Exchange Conference Proceedings[C]. Editorial Department of Acoustical Technology, 2019:4.

[6] Liu Kai, Wang Guan, Guo Jianzhong. Optimization Design of Matching Layer for Conave Spherical Ultrasonic Focusing Transducer [A]. Acoustical Society of China. Proceedings of the National Acoustics Congress 2019 [C]. Acoustical Society of China: Acoustical Society of China,2019:2.

[7] CHENG Jianzheng, ZHOU Yulu, MAO Yanxin, et al.Research on acoustic field characteristics of focusing transducer based on Rayleigh integral superposition method [J]. Chinese Journal of Computational Physics,2008(04):493-498.

[8] WANG Zhongwang, LI Hua, et al.Research on Numerical Simulation and Measurement of Sound Pressure of Focused Ultrasonic Vibrating Spherical Shell[J]. Mechanical and Electrical Engineering, 2019,36(09):949-953.

[9] Vivek T. Rathod. A Review of Acoustic Impedance Matching Techniques for Piezoelectric Sensors and Transducers[J]. Sensors,2020,20(14).

[10] Yinfang Jiang, Yongqiang Guo, Yulan Lei, Bo Chen, Bing Liu, Hongjin Kuang, Kaige Chen.Influence of matching layer thickness on performance of relaxation single crystal transducer [J]. Electronic Science and Technology,2018,31(09):25-28.

[11] TAN Bin, CHEN Yu, et al.Chinese Journal of Applied Mechanics,2012,29(01):32-37+115.

[12] He Yanbo, Gao Tianwen, et al.Study on Measurement Method of Oil Film Thickness Based on Phase Shift of Ultrasonic Reflection Coefficient [J]. Tribology, 201,41(01):1-8. 